\title{
Ovarian cancer biomarkers as diagnostic triage tests
}

This article was published in the following Dove Press journal:

Current Biomarker Findings

23 February 2013

Number of times this article has been viewed

\author{
Sara M Jordan \\ Robert E Bristow \\ Division of Gynecologic Oncology, \\ University of California, Irvine Medical \\ Center, Orange, CA, USA
}

\begin{abstract}
Ovarian cancer survival improves with accurate surgical staging, maximal tumor removal, and appropriate adjuvant chemotherapy. Therefore, survival is higher among patients managed by a gynecologic oncologist trained in these surgical techniques. Unfortunately, identifying patients preoperatively for referral to a gynecologic oncologist is often challenging, given that there are no definitive noninvasive diagnostic tests to triage patients with an adnexal mass to a surgical subspecialist. Inaccurate preoperative diagnosis of an adnexal mass frequently results in either unnecessary surgery for a benign mass or inadequate surgical staging for a malignant mass, with a subsequent negative effect on overall survival. Several recent tests have been investigated to improve the preoperative diagnosis of women presenting with adnexal masses. Cancer antigen 125 is the most commonly used serum marker for screening and monitoring of ovarian cancer, but is elevated in many benign conditions and falsely normal in $50 \%$ of early-stage epithelial ovarian cancers. The relatively low sensitivity and specificity of CA125 has driven researchers to identify new biomarkers and algorithms to assist with triaging adnexal masses. A promising new biomarker, human epididymis protein 4 , has been developed to monitor for recurrence of ovarian cancer. Three algorithms have also been developed, ie, risk of malignancy index, risk of ovarian malignancy algorithm, and OVA-1, which is the first diagnostic algorithm that combines multiple biomarkers for the purpose of triaging adnexal masses to be approved by the US Food and Drug Administration.
\end{abstract}

Keywords: ovarian cancer, biomarkers, CA125, RMI, ROMA, HE4, OVA-1

\section{Introduction}

The American Cancer Society has estimated that 22,280 women in the US will be diagnosed with ovarian cancer in 2012 and that 15,500 women will die from this disease according to the Surveillance Epidemiology and End Results database. ${ }^{1}$ Ten percent of American women undergo surgery during their lifetime for an adnexal mass, ${ }^{2}$ resulting in over 200,000 exploratory surgeries annually, and $13 \%-21 \%$ of these masses are found to be malignant. ${ }^{3}$ Despite extensive research to improve the diagnosis of ovarian cancer, this disease continues to be diagnosed at a late stage (Federation of Gynecology and Obstetrics III-IV) in $65 \%$ of cases.

Unfortunately, only $30 \%-50 \%$ of women with ovarian cancer are appropriately referred preoperatively to gynecologic oncologists for complete staging and maximal cytoreduction (tumor debulking). ${ }^{3}$ This is problematic because overall survival is inversely correlated with the amount of residual tumor up to a certain threshold frequently defined as residual tumor nodules each with no more than one centimeter maximal diameter. ${ }^{2,4,5}$ Complete surgical staging and cytoreduction requires specialized
Correspondence: Sara Jordan Division of Gynecologic Oncology, UC Irvine Medical Center, I0I The City Drive, Building 56, Room 260, Orange, CA 92868, USA

Tel + I 7144568020

Fax +I 7144566622

Email sara.jordan@uci.edu 
procedures that may include pelvic and para-aortic lymph node dissection, omentectomy, bowel resection, diaphragm resection, splenectomy, and complex tumor debulking. Accurate staging is critical in order to identify patients who need adjuvant treatment and to prevent overtreatment of patients who can be followed with surveillance alone.

Referral to a gynecologic oncologist is important in order to achieve complete surgical staging and maximal cytoreduction and to provide appropriate chemotherapy. The goal of primary surgery for advanced-stage ovarian cancer is to achieve no visible disease. If complete cytoreduction is unlikely to be achieved primarily, patients are offered neoadjuvant chemotherapy with the goal of reducing tumor burden to a resectable state and then performing interval maximal cytoreduction. Chemotherapy is tailored to an individual to achieve maximal benefit with minimal toxicity. There are many different cytotoxic and biochemical agents available and different methods of delivering the treatment, including intravenous or intraperitoneal delivery with varying dosing and scheduling. Therefore, a trained gynecologic oncologist should manage this complex disease.

The main challenge to identifying patients with high-risk adnexal masses preoperatively and correctly is the lack of definitive noninvasive diagnostic tests. Traditionally, cancer antigen (CA) 125 has been used as the standard serum marker to evaluate an adnexal mass. Unfortunately, CA125 is not an ideal test due to a relatively low sensitivity, specificity, and positive predictive value. Nevertheless, the current criteria recommended by the Society of Gynecologic Oncology and the American College of Obstetrics and Gynecology for referral to a gynecologic oncologist is as follows: postmenopausal women with CA125 above normal, premenopausal women with CA125 above $200 \mathrm{U} / \mathrm{mL}$, nodular or fixed pelvic mass, metastatic disease, ascites, or family history of breast or ovarian cancer. ${ }^{6}$

A recent study by investigators from the Mayo Clinic evaluated over 800 patients using the Society of Gynecologic Oncology and American College of Obstetrics and Gynecology referral guidelines for patients with an adnexal mass including CA125 in addition to menopausal status, imaging and examination findings, and family history and concluded that the guidelines performed well for advanced-stage ovarian cancer, but performed poorly for identifying earlystage ovarian cancer, especially in premenopausal women. Looking at all stages, the guidelines were $93.2 \%$ sensitive and $59.9 \%$ specific for postmenopausal women, with a positive predictive value of $64.6 \%$. Premenopausal women had a lower sensitivity of $79.2 \%$, a specificity of $69.8 \%$, and a positive predictive value of $39.6 \%$. The guidelines were created to ensure that women with advanced ovarian cancer were correctly referred preoperatively to a gynecologic oncologist. Unfortunately, the guidelines frequently miss early-stage disease. In premenopausal women, sensitivity is $92.3 \%$ for late-stage disease and $55.6 \%$ for early-stage disease. In postmenopausal women, sensitivity is $98.3 \%$ for late-stage and $79.6 \%$ for early-stage disease. ${ }^{7}$ For this reason, there is a need for diagnostic tests to improve appropriate preoperative referral of women, especially premenopausal women with early-stage ovarian cancer, to a gynecologic oncologist.

Because of the limitation in standard triage criteria and testing, recent efforts have focused on the development of novel serum biomarkers and triage algorithms using these markers in an attempt to improve the accuracy of referral and maximize the proportion of women with ovarian cancer that are appropriately referred to specialty care. Novel biomarkers and algorithms have been developed for improving the triage of adnexal masses. These include the risk of malignancy index (RMI), human epididymis protein 4 (HE4, the Architect HE4 Test, Abbott Laboratories, Abbott Park, IL), risk of ovarian malignancy algorithm (ROMA), and OVA-1 (Vermillion Inc, Austin, TX and Quest Diagnostics Inc, Madison, NJ). A comparison of the tests with regard to properties, cost, and ease of use is given in Table 1.

\section{Cancer antigen I 25}

CA125 is the most frequently ordered biomarker for triaging adnexal masses and is still believed by many to be the best single marker for diagnosing and monitoring ovarian cancer. CA125 is expressed by epithelial ovarian cancer and is not expressed by the surface epithelium of normal ovaries.

The sensitivity and specificity of CA125 for detecting ovarian cancer varies among different studies. Maggino et al examined the sensitivity and specificity of CA125 at various cutoffs. ${ }^{8}$ At a cutoff of $35 \mathrm{U} / \mathrm{mL}$, CA125 had a sensitivity of $78.3 \%$ and specificity of $82 \%$. Increasing the cutoff to $65 \mathrm{U} / \mathrm{mL}$, the sensitivity decreased to $71.7 \%$ and specificity increased to $92.5 \%$. A recent study published in $2011^{9}$ found that using a CA 125 cutoff of $88 \mathrm{U} / \mathrm{mL}$, sensitivity was $88 \%$, specificity was $97 \%$, the positive predictive value was $84 \%$, and the negative predictive value was $99 \%$. The reference value for CA125 depends on the laboratory and is frequently reported to be normal if less than $21 \mathrm{U} / \mathrm{mL}$ or less than $35 \mathrm{U} / \mathrm{mL}$.

A major diagnostic challenge is that CA125 lacks specificity and is associated with a high false positive rate. CA125 may be elevated in many benign processes, including 
Table I Comparison of CA I25, risk of malignancy index, risk of ovarian malignancy algorithm, human epididymis protein 4, OVA-I

\begin{tabular}{|c|c|c|c|c|c|}
\hline & CAI 25 & RMI (cutoff 200) & ROMA & HE4 & OVA-I \\
\hline \multicolumn{6}{|c|}{ Triage test $\mathrm{t}^{2,3,16,18-22,42}$} \\
\hline \multirow[t]{2}{*}{ Sensitivity (\%) } & $72-88$ & $7 I-92$ & 53-73 (premenopausal) & $93-94$ & $85-99$ \\
\hline & & & 83-9I (postmenopausal) & & \\
\hline \multirow[t]{2}{*}{ Specificity (\%) } & $62-78$ & $74-97$ & 74-88 (premenopausal) & $63-75$ & $28-51$ \\
\hline & & & 66-85 (postmenopausal) & & \\
\hline \multirow[t]{2}{*}{ PPV (\%) } & $52-72$ & $80-96$ & 34 (premenopausal) & & $40-91$ \\
\hline & & & 74 (postmenopausal) & & \\
\hline \multirow[t]{2}{*}{ NPV (\%) } & $78-87$ & $78-90$ & 95 (premenopausal) & & $93-97$ \\
\hline & & & 93 (postmenopausal) & & \\
\hline Cost $(\$)$ & 100 & Variable & 200 & 100 & 650 \\
\hline Ease & Serum only & Ultrasound required & Serum only, calculate & Serum only & Serum only \\
\hline
\end{tabular}

Abbreviations: PPV, positive predictive value; NPV, negative predictive value; RMI, risk of malignancy index; ROMA, risk of ovarian malignancy algorithm; HE4, human epididymis protein 4.

endometriosis, pelvic inflammatory disease, pregnancy, and diverticulitis. CA125 also lacks sensitivity, so although CA125 is appropriately elevated in $90 \%$ of advanced epithelial ovarian cancers, it is unfortunately not elevated in $50 \%$ of patients who have early-stage ovarian cancer. ${ }^{2,10-12}$

It is important to note that the only indication for CA125 testing approved by the US Food and Drug Administration (FDA) is monitoring for recurrence of ovarian cancer. Even a slight trend in rising CA125 can signify recurrent cancer. However, CA125 is often ordered for non-FDA approved indications (eg, screening or diagnostic work-up of an adnexal mass). In a study evaluating the indications for ordering CA125 tests, only $18 \%$ of patients had CA125 ordered for monitoring ovarian cancer, while $82 \%$ of patients had CA 125 ordered for non-FDA indications, including $15 \%$ of patients getting CA125 for preoperative evaluation of an adnexal mass and $67 \%$ of patients had CA125 inappropriately ordered for indications such as general screening. ${ }^{13}$

The use of CA125 and transvaginal ultrasound for screening the general population was studied in the Prostate, Lung, Colorectal, and Ovarian Screening Trial presented at the American Society of Clinical Oncology in 2011. ${ }^{14}$ This was a randomized, multicenter study of 78,216 asymptomatic women in the general population aged 55-74 years between 1993 to 2001 who were randomized to undergo either annual screening with CA125 and transvaginal ultrasound or usual care and followed for diagnosis of ovarian cancer until 2010. This study concluded that screening with CA125 and transvaginal ultrasound does not decrease the risk of dying from ovarian cancer and unnecessarily increases the number of false positives and unwarranted follow-up procedures. Therefore, CA125 is not used for screening the general population, but rather for diagnostic purposes for a known mass or surveillance of a known malignancy.

\section{Risk of malignancy index}

The RMI was developed with the goal of identifying patients at high risk of harboring an ovarian malignancy in order to triage patients accurately to specialty care. First described by Jacobs et al in 1990, RMI is the product of CA125 (U/mL), the ultrasound result (expressed as $0,1,3$ or 4), and menopausal status ( 1 if premenopausal and 3 if postmenopausal). ${ }^{15}$ This study concluded that RMI was effective for discriminating between malignant and benign adnexal masses, with a sensitivity of $85 \%$ and specificity of $97 \%$ using an RMI cutoff of 200 . RMI over 200 was associated with 42 times the risk of malignancy, while values less than 200 corresponded to 0.15 times the risk. RMI was adjusted by Tingulstad et $\mathrm{al}^{16}$ in 1996 (RMI II) and again in 1999 (RMI III). ${ }^{17}$ Ultrasound imaging was scored as one point for each of the following characteristics: septations, solid areas, metastatic disease, ascites, and bilateral lesions. The difference in the three scores is based on how $\mathrm{U}$ (ultrasound score) and $\mathrm{M}$ (menopausal status) are assigned:

- RMI $1=\mathrm{U} \times \mathrm{M} \times \mathrm{CA} 125$; ultrasound score of 0 is $\mathrm{U}=0$, score of 1 is $\mathrm{U}=1$, and a score of $\geq 2$ is $\mathrm{U}=3$. Premenopausal is $\mathrm{M}=1$ and postmenopausal is $\mathrm{M}=3$.

- $\quad$ RMI II $=\mathrm{U} \times \mathrm{M} \times \mathrm{CA} 125$; ultrasound score of 0 or 1 is $\mathrm{U}=1$, and score of $\geq 2$ is $\mathrm{U}=4$. Premenopausal is $\mathrm{M}=1$ and postmenopausal is $\mathrm{M}=4$.

- $\quad$ RMI III $=\mathrm{U} \times \mathrm{M} \times \mathrm{CA} 125$; ultrasound score of 0 or 1 is $\mathrm{U}=1$, and score of $\geq 2$ is $\mathrm{U}=3$. Premenopausal is $\mathrm{M}=1$ and postmenopausal is $\mathrm{M}=3$.

Several studies have compared these scoring schemes. Initial studies reported a higher specificity of RMI II compared with RMI I, ${ }^{16,18,19}$ but a more recent study published in $2011^{9}$ compared these three indices and CA125 in 182 women, and concluded that there was no statistically significant difference between the three scoring algorithms and CA125 in differentiating between benign and malignant adnexal masses. 
A comprehensive meta-analysis evaluating prediction models for preoperative assessment of adnexal masses was published in 2009. ${ }^{20}$ This paper identified 1277 publications and focused on 109 studies that met selection criteria. RMI I and RMI II were found to be the best predictors. When $\mathrm{RMI}=200$ was used, the sensitivity was $78 \%$ and specificity $87 \%$ for detecting an ovarian malignancy. This large metaanalysis concluded that RMI should be the model of choice for preoperative assessment of adnexal masses.

Several other studies have published similar results advocating the use of RMI to triage adnexal masses. A Danish prospective study enrolled 1159 patients who were referred to a tertiary center including 251 patients with ovarian cancer and 74 patients with borderline tumors. Sensitivity and specificity of RMI were $92 \%$ and $82 \%$, respectively. ${ }^{21}$ A prospective multicenter Dutch study enrolled 548 women with adnexal masses and concluded that sensitivity was $81 \%$ and specificity $85 \%$, with a positive predictive value of $48 \%$ and a negative predictive value of $96 \%$. In The Netherlands at the time of this study, only $64 \%$ of patients with ovarian cancer were being operated on by a gynecologic oncologist, and this would have increased to $80 \%$ if an RMI cutoff of 200 had been used. ${ }^{21,22}$ In Norway, 173 women were enrolled between 1992 and 1994 and using a RMI cutoff of 200, sensitivity was $80 \%$ and specificity was $92 \% .{ }^{16}$ A Brazilian study enrolled 158 women between 1996 and 1998 and, using a lower RMI cutoff of 150 , sensitivity and specificity were both $79 \%{ }^{23}$ In general, the quoted sensitivity of RMI is $71 \%-88 \%$ and specificity is $74 \%-97 \%{ }^{3}$

RMI is an accurate predictor of malignancy, and is reliable, cheap, readily available, and cost-effective. ${ }^{24}$ RMI has the potential to reduce the number of benign procedures performed when CA125 is used alone due to the high false positive rate of CA125..$^{25}$ One of the main limitations of RMI is that the relatively low prevalence of ovarian cancer in the general population decreases performance of this test. Another limitation is that the RMI algorithm relies on subjective interpretation of ultrasound imaging, which is highly variable. In practice, this test is rarely used and is replaced with tests that offer objective methods of quantifying the risk of malignancy for adnexal masses.

\section{Human epididymis protein 4}

HE4, a promising new biomarker, is a glycoprotein overexpressed by epithelial ovarian cancer. It is overexpressed in $93 \%$ of serous, $100 \%$ of endometrioid, and $50 \%$ of clear cell (not mucinous) ovarian carcinomas. It was originally part of a panel of nine biomarkers studied in 1999 and was found to be the marker most highly overexpressed in ovarian cancer. ${ }^{26}$ The normal reference range of HE4 is less than $151 \mathrm{pM}$. Both the sensitivity and specificity of HE4 for epithelial ovarian cancer are relatively high at $93 \%-94 \%$ and $75 \%$, respectively. ${ }^{3}$ The HE4 enzyme immunoassay test kit (Fujirebio Diagnostics, Göteborg, Sweden) was cleared by the US FDA in 2008 and has been approved for monitoring recurrence or progression of epithelial ovarian cancer. In 2010, the Architect HE4 (Abbott Diagnostics UK, Maidenhead, UK codeveloped with Fujirebio Diagnostics), an automated version of the HE4 enzyme immunoassay test, was approved by the FDA for the same indications.

Several studies have reported that HE4 is a better marker compared with CA125 for diagnosing ovarian cancer. ${ }^{27,28}$ In a study by Moore et al, ${ }^{27}$ preoperative CA125 and HE4 were collected from 233 patients undergoing surgery for an adnexal mass. As a single biomarker, HE4 had the highest sensitivity of $72.9 \%$ (specificity $95 \%$ ) for detecting ovarian cancer, especially stage I disease. Holcomb et al recently published a study comparing HE4 and CA125 specifically in 229 premenopausal women..$^{28}$ In this population, $85 \%$ of women had benign disease, $8 \%$ had epithelial ovarian cancer, and 7\% had borderline tumors. The sensitivity of CA125 and HE4 for epithelial ovarian cancer detection was $83.3 \%$ and $88.9 \%$, respectively. The specificity of CA125 and HE4 was $59.5 \%$ and $91.8 \%$, respectively. Normal HE4 ruled out invasive cancer in $98 \%$ of women with an elevated CA125.

Higher sensitivity of HE4 compared with CA125 specifically in premenopausal women results in better detection of early-stage ovarian malignancies and borderline tumors. ${ }^{27}$ The higher specificity of HE4 results in fewer abnormal results for benign conditions and, for this reason, HE4 is frequently used for monitoring purposes in recurrent ovarian cancer. ${ }^{29}$ An increase in HE4 value of $25 \%$ higher than a prior value is considered to be a positive change, concerning for recurrence or progression because HE4 rises by at least $25 \%$ in $60 \%$ of women with ovarian cancer relapse or progression. HE4 remains constant in $75 \%$ of women without disease progression. For this reason, gynecologic oncologists are increasingly using HE4 specifically in the setting of monitoring for recurrence. Nonetheless, it is important to note that the concept of early detection of biochemical recurrence of ovarian cancer, without symptomatic disease progression, has been called into question. There is no clear evidence that initiating treatment at the time of biochemical recurrence significantly improves overall survival and, on the contrary, early treatment may negatively impact quality of life. 
However, other studies have found similar performance of HE4 and CA125 with regard to diagnostic utility in triaging adnexal masses. In a 2012 study of 1218 patients including 252 with ovarian cancer and 79 borderline ovarian tumors, specificity was $62.2 \%$ for CA125 and $63.4 \%$ for HE4 with a sensitivity of $94.4 \%{ }^{30} \mathrm{~A}$ retrospective study of 80 patients with ovarian cancer was presented at the American Society of Clinical Oncology in 2008. HE4 and CA125 had similar diagnostic potential, with HE4 correlating with clinical status in $76 \%$ of cases and CA25 correlating in $79 \%$ of cases. ${ }^{31}$ In general the two tests, CA125 and HE4, are felt to have similar diagnostic potential.

\section{Risk of ovarian cancer malignancy}

A more recently developed algorithm reported by Moore et al in 2009 is the risk of ovarian cancer malignancy (ROMA) that combines CA125 and HE4 and also takes into account menopausal status without relying on imaging findings. ${ }^{2}$ A ROMA value is obtained using an online calculator that combines menopausal status, CA125, and HE4 levels. The objective of this study was to report the predictive value of ROMA to assess the risk of ovarian cancer in women with a pelvic mass. In this study, 12 sites enrolled 531 premenopausal and postmenopausal women who were tested preoperatively for CA125 and HE4. There were 352 benign tumors, 129 epithelial ovarian cancers, 22 low malignant potential tumors, six nonepithelial ovarian cancers, and 22 nonovarian cancers. ROMA stratified patients according to high risk and low risk, and correctly classified $93.8 \%$ of patients with epithelial ovarian cancer as being at high risk. In the postmenopausal group, sensitivity for epithelial ovarian cancer was $92.3 \%$ and specificity was $74.8 \%$. In the premenopausal group, sensitivity was much lower at $76.5 \%$ and specificity $74.8 \%$. This low sensitivity in premenopausal patients is one of the main shortcomings of ROMA. Another limitation of ROMA is the lower performance of this test in patients with early-stage compared with later-stage ovarian cancer. Of the 129 patients with ovarian cancer, eight were misdiagnosed using ROMA, including 5/8 (63\%) who were stage I/II and only $1 / 8(13 \%)$ who were stage III/IV, and two patients were unstaged. Furthermore, of the patients misclassified as being at low risk, half of them had tumors of low malignant potential. Staging low malignant potential tumors is debatable, yet misdiagnosis of these patients preoperatively hinders the opportunity to stage patients who may otherwise have undergone further staging.

In a prospective, multicenter National Institutes of Health trial, Moore et al enrolled 531 women and found that ROMA successfully identified $93.8 \%$ of patients with epithelial ovarian cancer as being "high-risk" and identified $75 \%$ of patients with benign disease as "low-risk", concluding that ROMA is superior to RMI. However, ROMA was not as accurate in predicting all-cause malignancy because $21 \%(3 / 14)$ of patients with nongynecologic malignancies and 25\% (2/8) with other gynecologic malignancies were misdiagnosed as being at low risk. ${ }^{2}$ In a multicenter trial that enrolled 457 women, ROMA was compared with RMI, and ROMA had a higher sensitivity (94.3\%) compared with RMI (84.6\%) at a set specificity of $75 \% .{ }^{32}$ For early-stage disease, ROMA had a sensitivity of $85.3 \%$ whereas RMI had a sensitivity of $64 \%$.

The 2012 Danish Pelvic Mass study concluded that ROMA performs as well as RMI without the additional need for potentially subjective ultrasound evaluation. This was a large prospective study of 1218 women presenting with a pelvic mass and compared CA125, RMI, HE4, and ROMA. ${ }^{30}$ At a set specificity of $75 \%$, RMI had a slightly higher sensitivity of $96 \%$ compared with the ROMA sensitivity of $94.8 \%$. For a set sensitivity of $94.4 \%$ (corresponding to RMI 200), CA125 had a sensitivity of $62.2 \%$, HE4 63.2\%, ROMA $76.5 \%$, and RMI 81.5\%. Therefore, the authors of this study recommended that ROMA should be the first-line test for selecting high-risk patients for referral to a surgeon trained in oncology.

ROMA was also compared with CA125 in a 2011 study of 519 women with either ovarian cancer or a benign adnexal mass, or healthy women without any mass, and CA125 was still the best single marker to diagnose malignancy, but ROMA (with the addition of HE4) improved the diagnostic accuracy. $^{33}$

Several studies have compared the diagnostic potential of ROMA between premenopausal and postmenopausal patients. A study of 104 Italian women with a pelvic mass concluded that ROMA performed highest in postmenopausal women but remained a challenge in premenopausal women. In the premenopausal group, there were 15 epithelial ovarian cancers, eight of which were correctly classified by ROMA as high-risk, with a sensitivity of $53.3 \%$. In the postmenopausal group, there were 40 epithelial ovarian cancers, 33 of which were classified by ROMA as high-risk with a sensitivity of $82.5 \% .{ }^{34}$ On the other hand, a Serbian study of 108 women compared ROMA between premenopausal and postmenopausal women and found that ROMA correctly classified 96\% of premenopausal women with a benign mass as being low risk. They concluded that ROMA is a useful test for excluding malignancy not only in postmenopausal women, but in 
premenopausal women as well. ${ }^{35}$ This was a small study and concern remains about poorer diagnostic potential of ROMA specifically among premenopausal patients.

Several studies have compared ROMA with CA125 and HE4 alone and concluded that ROMA may not be superior to either biomarker alone. A 2011 study of 527 patients including 111 ovarian cancers concluded that, in cases where CA125 and HE4 are both known, ROMA is most useful when CA125 is elevated and HE4 is normal. When HE4 was elevated, addition of ROMA did not increase sensitivity and only increased specificity by $3.2 \%$ compared with HE4 alone. ${ }^{36}$ A study of 104 patients with a pelvic mass, including 55 epithelial ovarian cancers, concluded that ROMA had excellent diagnostic potential, especially in postmenopausal women, but ROMA was not superior to HE4 alone. In this study, the calculated area under the curve for HE4 in postmenopausal women was slightly higher (0.94) than for ROMA (0.92) and was essentially the same in premenopausal women (0.77). ${ }^{34}$ Similarly, a prospective validation study concluded that HE4 was superior to CA125, but there is no benefit in combining both markers using ROMA in the clinical setting. Of the 160 patients included in this study, there were 56 borderline tumors and cancers in general which included 29 epithelial ovarian cancers. HE4 and ROMA had a similar area under the curve (0.86 and 0.87 ), specificity (85.9\% and $87.3 \%$ ), and sensitivity (78.9\% and 78.9\%, respectively). Therefore, there was no benefit found for using ROMA compared with HE4 alone. ${ }^{37}$

\section{OVA-I}

OVA-1 is a relatively new and promising diagnostic algorithm to triage women aged 18 years and older with a pelvic mass and a plan to proceed with surgery. This test is used by general gynecologists as a triage tool to determine the need for gynecologic oncology referral. OVA-1 combines imaging, menopausal status, CA125, and four other biomarkers, ie, prealbumin, apolipoprotein A-1, $\beta 2$-microglobulin, and transferrin. ${ }^{38}$ Approved by the US FDA in September 2009, OVA-1 is the first diagnostic algorithm that combines multiple biomarkers. ${ }^{39}$ OVA-1 is a test that is ordered and the score is reported as a single result that claims to be more reliable than any of the individual biomarkers alone. The OVA-1 score is reported as a number between 1 and 10, and a value considered to reflect a high risk of malignancy is $\geq 4.4$ for postmenopausal women and $\geq 5$.0 for premenopausal women.

A prospective, multicenter, large-scale, pivotal, clinical trial published by Ueland et al evaluated 516 cases comparing physician assessment with OVA- $1 .{ }^{40}$ The addition of OVA-1 to physician assessment improved sensitivity from $72.2 \%$ to $91.7 \%$ for nongynecologic oncologists and from $77.5 \%$ to $98.9 \%$ for gynecologic oncologists. Addition of OVA-1 correctly identified malignancies missed by assessment alone in $70 \%$ of nongynecologic oncologists and $95 \%$ of gynecologic oncologists. OVA-1 detected $76 \%$ of ovarian cancers that were missed by CA125. OVA-1 was predictive for both early and late stage cancers.

A second prospective multicenter trial recently published by Bristow et al validated the effectiveness of OVA-1 to diagnose ovarian malignancy compared with clinical assessment and CA125 among women undergoing surgery for an adnexal mass initially evaluated by a nongynecologic oncologist. ${ }^{41}$ A total of 494 patients were enrolled including 65 women (13.2\%) who were diagnosed with an ovarian malignancy and $43.1 \%$ of whom had stage I disease. A sensitivity of $95.7 \%$ was reported for OVA-1 when combined with physician assessment. OVA-1 correctly predicted ovarian malignancy in $91.4 \%$ of early-stage disease, compared with only $65.7 \%$ using CA125. OVA-1 correctly identified $83.3 \%$ of malignancies that were missed by clinical impression and $70.8 \%$ missed by CA125. The high negative predictive value of OVA-1 was confirmed in this study as $98.1 \%$.

Bast et al recently reported a review of multiple biomarkers to triage adnexal masses ${ }^{3}$ and quoted the sensitivity of OVA-1 as $85 \%$ for premenopausal women and as high as $96 \%$ for postmenopausal women with specificity of $28 \%$ for premenopausal and $40 \%$ for postmenopausal women. OVA-1 also has a high negative predictive value of $94 \%-96 \%$ in lowrisk women, which makes this clinically useful in regards to triaging patients for referral to gynecologic oncologists. Due to increased sensitivity, OVA-1 may be elevated in situations where CA125 is normal and this leads to more referrals to gynecologic oncologists for patients who may have otherwise not been referred. Unfortunately, due to higher sensitivity with the goal of not underdiagnosing malignancy, there is also a resulting loss of specificity.

OVA-1 is useful for general gynecologists if there is equivocal concern for malignancy based on history, examination, and imaging. If a gynecologic oncologist plans to operate for this mass, then an OVA-1 does not need to be ordered preoperatively because the intraoperative findings and frozen section will determine if further staging is necessary. OVA-1 can be used in addition to history, examination, and imaging, but should never be used as a primary screening test alone. ${ }^{39}$ The main limitation of OVA-1 is the cost since a single test is $\$ 650$. However, this upfront 
financial cost must be balanced against the potential negative impact on patient care and clinical outcome for those patients ultimately diagnosed with ovarian cancer that did not receive standard surgical intervention resulting from failure to refer to a gynecologic oncologist.

\section{Other diagnostic biomarkers}

There are several other serum biomarkers that are useful for triaging patients with an adnexal mass. Carcinoembryonic antigen is a protein that may be elevated in mucinous ovarian cancers or gastrointestinal cancers as well as other malignancies that produce protein such as breast, pancreas, thyroid, and lung cancer. Carcinoembryonic antigen can also be elevated in benign conditions such as mucinous cystadenoma of the ovary, inflammatory bowel disease, pancreatitis, or pulmonary infections. Cancer antigen 19-9 is another mucin protein that is sometimes elevated in ovarian cancers, although this marker is used more commonly to monitor gastrointestinal and especially pancreatic and gallbladder malignancies. Cancer antigen 15-3 can similarly be useful to differentiate between gynecologic and gastrointestinal, pancreatic, or breast malignancies. Alfa-fetoprotein, lactate dehydrogenase, beta human chorionic gonadotropin, and inhibin aid with diagnosing ovarian germ cell malignancies. ${ }^{12}$

The use of biomarkers to triage adnexal masses continues to be an active area of research. Some biomarkers that have recently demonstrated potential include osteopontin, mesothelin, lysophosphatic acid, haptoglobin, and transthyretin. However, most experimental biomarkers remain unavailable for commercial use until further studies demonstrate clinical benefit such as the markers discussed in detail in this paper.

\section{Recommendations}

In light of the studies evaluating various biomarkers and algorithms to screen for and monitor recurrence of ovarian cancer, several recommendations are suggested. Work-up of an adnexal mass should always include a patient history including family history, physical examination, and imaging. For screening purposes, we recommend transvaginal ultrasound and possibly computed tomography if there is concern for metastatic disease. If a generalist is working up a patient, and the patient has imaging or clinical criteria for surgery, then OVA-1 is indicated to triage referral to a gynecologic oncologist. If a gynecologic oncologist sees the patient preoperatively, there is no need for an OVA-1 or ROMA, but a CA125 can be considered as a baseline level in the event that the patient is subsequently diagnosed with ovarian cancer.

\section{Disclosure}

The authors report no conflicts of interest in this work.

\section{References}

1. National Cancer Institute. Surveillance Epidemiology and End results. SEER Stat Fact Sheets: Ovary. Available from: http://seer.cancer.gov/ statfacts/html/ovary.html. Accessed January 11, 2013.

2. Moore RG, McMeekin DS, Brown AK, et al. A novel multiple marker bioassay utilizing HE4 and CA125 for the prediction of ovarian cancer in patients with a pelvic mass. Gynecol Oncol. 2009;112(1): $40-46$.

3. Bast RC, Skates S, Lokshin A, Moore RG. Differential diagnosis of a pelvic mass: improved algorithms and novel biomarkers. Int J Gynecol Cancer. 2012;22(10 Suppl 1):S5-S8.

4. Bristow RE, Montz FJ, Lagasse LD, Leuchter RS, Karlan BY. Survival impact of surgical cytoreduction in stage IV epithelial ovarian cancer. Gynecol Oncol. 1999;72(3):278-287.

5. Giede KC, Kieser K, Dodge J, Rosen B. Who should operate on patients with ovarian cancer? An evidence-based review. Gynecol Oncol. 2005;99(2):447-461.

6. Gostout BS, Brewer MA. Guidelines for referral of the patient with an adnexal mass. Clin Obstet Gynecol. 2006;49(3):448-458.

7. Dearking AC, Aletti GD, McGree ME, Weaver AL, Sommerfield MK, Cliby WA. How relevant are ACOG and SGO guidelines for referral of adnexal mass? Obstet Gynecol. 2007;110(4):841-848.

8. Maggino T, Gadducci A, D'Addario V, et al. Prospective multicenter study on CA125 in postmenopausal pelvic masses. Gynecol Oncol. 1994;54(2):117-123.

9. Bouzari Z, Yazdani S, Ahmadi MH, et al. Comparison of three malignancy risk indices and CA-125 in the preoperative evaluation of patients with pelvic masses. BMC Res Notes. 2011;4:206.

10. Li AJ. New biomarkers for ovarian cancer: OVA-1 and ROMA in diagnosis. Int J Gynecol Cancer. 2012;22 Suppl 1:S5-S8.

11. Zurawski VR, Knapp RC, Einhorn N, et al. An initial analysis of preoperative serum CA125 levels in patients with early stage ovarian carcinoma. Gynecol Oncol. 1988;30(1):7-14.

12. Tanyi JL, Scholler N. Oncology biomarkers for gynecologic malignancies. Front Biosci (Elite Ed). 2012;4:1097-1110.

13. Petignat $\mathrm{P}$, Joris F, Obrist R. How CA125 is used in routine clinical practice. Eur J Cancer. 2000;36(15):1933-1937.

14. Buys SS, Partridge E, Black A, et al. Effect of screening on ovarian cancer mortality: the Prostate, Lung, Colorectal and Ovarian (PLCO) cancer screening randomized controlled trial. JAMA. 2011;305(22): 2295-2303.

15. Jacobs I, Oram D, Fairbanks J, Turner J, Frost C, Grudzinskas JG. A risk of malignancy index incorporating CA125, ultrasound and menopausal status for the accurate preoperative diagnosis of ovarian cancer. $\mathrm{Br} \mathrm{J}$ Obstet Gynaecol. 1990;97(10):922-929.

16. Tingulstad S, Hagen B, Skjeldestad FE, et al. Evaluation of a risk of malignancy index based on serum CA125, ultrasound findings and menopausal status in the pre-operative diagnosis of pelvic masses. $\mathrm{Br}$ J Obstet Gynaecol. 1996;103(8):826-831.

17. Tingulstad S, Hagen B, Skjeldestad FE, Halvorsen T, Nustad K, Onsrud M. The risk-of-malignancy index to evaluate potential ovarian cancers in local hospitals. Obstet Gynecol. 1999;93(3):448-452.

18. Morgante G, la Marca A, Ditto A, De Leo V. Comparison of two malignancy risk indices based on serum CA125, ultrasound score and menopausal status in the diagnosis of ovarian masses. Br J Obstet Gynaecol. 1999;106(6):524-527.

19. Aslam N, Tailor A, Lawton F, Carr J, Savvas M, Jurkovic D. Prospective evaluation of three different models for the pre-operative diagnosis of ovarian cancer. BJOG. 2000;107(11):1347-1353.

20. Geomini P, Kruitwagen R, Bremer GL, Cnossen J, Mol BW. The accuracy of risk scores in predicting ovarian malignancy: a systematic review. Obstet Gynecol. 2009;113(2 Pt 1):384-394. 
21. Håkansson F, Høgdall EV, Nedergaard L, et al. Risk of malignancy index used as a diagnostic tool in a tertiary centre for patients with a pelvic mass. Acta Obstet Gynecol Scand. 2012;91(4):496-502.

22. van den Akker PA, Aalders AL, Snijders MP, et al. Evaluation of the Risk of Malignancy Index in daily clinical management of adnexal masses. Gynecol Oncol. 2010;116(3):384-388.

23. Torres JC, Derchain SF, Faundes A, Gontijo RC, Martinez EZ, Andrade LA. Risk-of-malignancy index in preoperative evaluation of clinically restricted ovarian cancer. Sao Paulo Med J. 2002;120(3): 72-76.

24. Enakpene CA, Omigbodun AO, Goecke TW, Odukogbe AT, Beckmann MW. Preoperative evaluation and triage of women with suspicious adnexal masses using risk of malignancy index. J Obstet Gynaecol Res. 2009;35(1):131-138.

25. Raza A, Mould T, Wilson M, Burnell M, Bernhardt L. Increasing the effectiveness of referral of ovarian masses from cancer unit to cancer center by using a higher referral value of the risk of malignancy index. Int J Gynecol Cancer. 2010;20(4):552-554.

26. Schummer M, Ng WV, Bumgarner RE, et al. Comparative hybridization of an array of 21,500 ovarian cDNAs for the discovery of genes overexpressed in ovarian carcinomas. Gene. 1999;238(2): 375-385.

27. Moore RG, Brown AK, Miller MC, et al. The use of multiple novel tumor biomarkers for the detection of ovarian carcinoma in patients with a pelvic mass. Gynecol Oncol. 2008;108(2):402-408.

28. Holcomb K, Vucetic Z, Miller MC, Knapp RC. Human epididymis protein 4 offers superior specificity in the differentiation of benign and malignant adnexal masses in premenopausal women. Am J Obstet Gynecol. 2011;205(4):358. e1-e6.

29. Langmár Z, Németh M, Vleskó G, Király M, Hornyák L, Bösze P. HE4 - a novel promising serum marker in the diagnosis of ovarian carcinoma. Eur J Gynaecol Oncol. 2011;32(6):605-610.

30. Karlsen MA, Sandhu N, Høgdall C, et al. Evaluation of HE4, CA125, risk of ovarian malignancy algorithm (ROMA) and risk of malignancy index (RMI) as diagnostic tools of epithelial ovarian cancer in patients with a pelvic mass. Gynecol Oncol. 2012;127(2):379-383.

31. Allard J. Use of a novel biomarker HE4 for monitoring patients with epithelial ovarian cancer. J Clin Oncol. 2009;26 Suppl:abstr 5535.
32. Moore RG, Jabre-Raughley M, Brown AK, et al. Comparison of a novel multiple marker assay vs the Risk of Malignancy Index for the prediction of epithelial ovarian cancer in patients with a pelvic mass. Am J Obstet Gynecol. 2010;203(3):228. e1-e6.

33. Lenhard M, Stieber P, Hertlein L, et al. The diagnostic accuracy of two human epididymis protein 4 (HE4) testing systems in combination with CA125 in the differential diagnosis of ovarian masses. Clin Chem Lab Med. 2011;49(12):2081-2088.

34. Montagnana M, Danese E, Ruzzenente O, et al. The ROMA (Risk of Ovarian Malignancy Algorithm) for estimating the risk of epithelial ovarian cancer in women presenting with pelvic mass: is it really useful? Clin Chem Lab Med. 2011;49(3):521-525.

35. Kadija S, Stefanovic A, Jeremic K, et al. The utility of human epididymal protein 4, cancer antigen 125 , and risk for malignancy algorithm in ovarian cancer and endometriosis. Int J Gynecol Cancer. 2012;22(2): 238-244.

36. Molina R, Escudero JM, Augé JM, et al. HE4 a novel tumour marker for ovarian cancer: comparison with CA125 and ROMA algorithm in patients with gynaecological diseases. Tumour Biol. 2011;32(6): 1087-1095.

37. Jacob F, Meier M, Caduff R, et al. No benefit from combining HE4 and CA125 as ovarian tumor markers in a clinical setting. Gynecol Oncol. 2011;121(3):487-491.

38. Zhang Z. An in vitro diagnostic multivariate index assay (IVDMIA) for ovarian cancer: harvesting the power of multiple biomarkers. Rev Obstet Gynecol. 2012;5(1):35-41.

39. Muller CY. Doctor, should I get this new ovarian cancer test-OVA-1? Obstet Gynecol. 2010;116(2 Pt 1):246-247.

40. Ueland FR, Desimone CP, Seamon LG, et al. Effectiveness of a multivariate index assay in the preoperative assessment of ovarian tumors. Obstet Gynecol. 2011;117(6):1289-1297.

41. Bristow RE, Smith A, Zhang Z, et al. Ovarian malignancy risk stratification of the adnexal mass using a multivariate index assay. Gynecol Oncol. 2012;pii:S0090-8258(12)00888-00888.

42. Ware Miller R, Smith A, DeSimone CP, et al. Performance of the American College of Obstetricians and Gynecologists' ovarian tumor referral guidelines with a multivariate index assay. Obstet Gynecol. 2011;117(6):1298-1306.
Current Biomarker Findings

\section{Publish your work in this journal}

Current Biomarker Findings is an international, peer-reviewed, open access journal publishing original research, reports, reviews and commentaries on all areas of biomarker research. The manuscript management system is completely online and includes a very quick and fair

\section{Dovepress}

peer-review system. Visit http://www.dovepress.com/testimonials.php to read real quotes from published authors. 